\title{
Distribution pattern of lysosomal granules in fibroblasts of the Chediak-Higashi syndrome
}

\author{
K ABE, S ARASHIMA, M HONMA \\ From the Department of Anatomy and Pediatrics, Hokkaido University School of Medicine, Sapporo, \\ Japan
}

SUMMARY Cultured fibroblasts from a patient with the Chediak-Higashi syndrome, the mother of the patient, and a normal control were studied by light and electron microscopy. The distribution pattern of PAS-positive and acid phosphatase-containing granules in the cytoplasm differed significantly in the fibroblasts from the patient when compared with those from the mother and control. The granules in the fibroblasts from the patient were clustered in the perinuclear area, whereas the granules in the fibroblasts from the mother and control were dispersed throughout the cytoplasm. After incubation with ascorbic acid, the clustered granules in the fibroblasts of the Chediak-Higashi syndrome showed a tendency to spread throughout the cytoplasm. The distribution pattern of the granules was studied by quantitative morphology.

The Chediak-Higashi syndrome is a rare genetic disorder characterised by the presence of abnormally large inclusions in polymorphonuclear leucocytes. ${ }^{1-3}$ Certain mutants of Aleutian minks and beige mice are known to be homologues of the Chediak-Higashi syndrome in humans. ${ }^{45}$ Although the pathogenesis of this syndrome remains uncertain, recently it has been reported that the Chediak-Higashi syndrome shows abnormal function of microtubules, ${ }^{6}$ and the function is restored by ascorbic acid. ${ }^{7}$ The pathogenesis of some genetic disorders has been explained with cell culture. ${ }^{8-10}$ Abnormally large inclusions have been observed in cultured fibroblasts from a patient with the Chediak-Higashi syndrome ${ }^{11}$ and beige mice. ${ }^{6}$ Thus, a culture of the fibroblasts derived from the patient with the Chediak-Higashi syndrome may be useful for understanding its pathogenesis.

We recently had a patient with the ChediakHigashi syndrome. ${ }^{12}$ The case showed abnormal inclusions in the polymorphonuclear leucocytes and lymphocytes, and died 110 days after birth. From skin biopsy of the patient, we established the cell lines of fibroblasts, and the cultured fibroblasts showed characteristic distribution pattern of lysosomal granules. In this study, the cultured skin fibroblasts of the Chediak-Higashi syndrome, and the effects of ascorbic acid on the fibroblasts were examined cytologically.

\section{Material and methods}

Skin fibroblasts from a 95-day-old girl with the Chediak-Higashi syndrome, a 26-year-old mother of the patient, and a 37-year-old healthy male as a control were cultured in monolayer on cover slips. The fibroblasts were grown in Eagle's medium with $15 \%$ calf serum. Eight different subcultures out of 26 subcultures in each cell line were examined at the second day of the culture by light and electron microscopy.

Fibroblasts on the cover glasses were fixed with $2 \%$ glutaraldehyde in $0.1 M$ cacodylate buffer ( $\mathrm{pH} \mathrm{7.4)}$ at $4^{\circ} \mathrm{C}$ for between $30 \mathrm{~min}$ and two hours. For light microscopy, they were stained with periodic acidSchiff reagent (PAS) and haematoxylin, or reacted by Gomori's method for acid phosphatase. Some of them were stained with Giemsa, toluidine blue, or Sudan black B. For electron microscopy, after fixation with glutaraldehyde, they were fixed with $2 \%$ $\mathrm{OsO}_{4}$ in $0.1 M$ cacodylate buffer $(\mathrm{pH} 7.4)$ at $4{ }^{\circ} \mathrm{C}$ for one hour and embedded in Epon. Ultrathin sections were stained with uranyl acetate and lead citrate, and examined by electron microscopy.

\section{Effects of ascorbic acid}

Fibroblasts from the patient, mother of the patient, and the control were incubated in the medium containing $0 \cdot 1,1,10$, or $100 \mathrm{~m} M$ ascorbic acid for $15 \mathrm{~min}$ before fixation and examined by light microscopy. Two cultures for each source were examined. 


\section{Results}

The cultured fibroblasts showed no significant differences in cellular shape and number for the cultures derived from the patient with the ChediakHigashi syndrome, the mother of the patient, and the healthy control (Figs. 1-3). They were spindle-

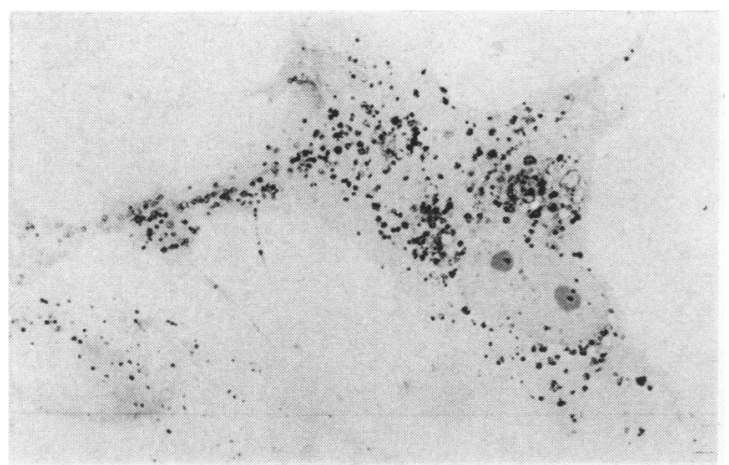

Fig. 1 Cultured fibroblast from the control. Lysosomal granules are dispersed throughout the cytoplasm. Acid phosphatase demonstrated by Gomori's method. Haematoxylin $\times 700$.

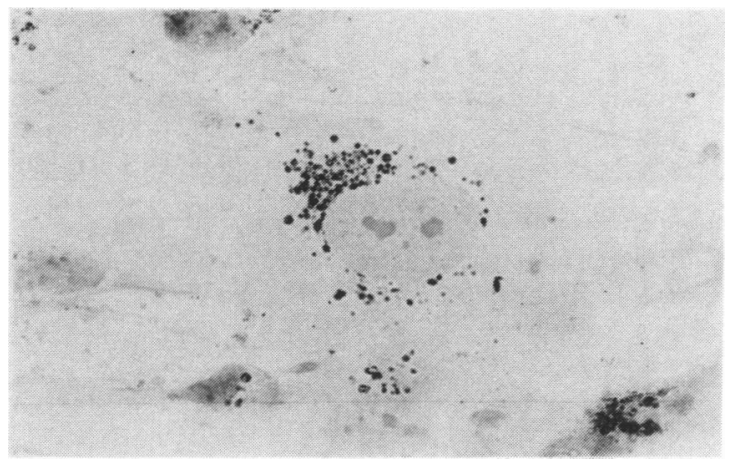

Fig. 2 Cultured fibroblast from the patient with Chediak-Higashi syndrome. Lysosomal granules are clustered adjacent to the nucleus. Gomori's method. Haematoxylin $\times 700$.

shaped or stellate with a few cytoplasmic processes, and the nuclei were oval and had several nucleoli. The cytoplasm contained a large number of small granules which were PAS-positive and showed acid phosphatase activity (Figs. 1-3). These granules were not stained with Giemsa, toluidine blue, or Sudan black B. The distribution of these cytoplasmic granules in the fibroblasts, however, exhibited significant differences between the fibroblasts derived from the patient and those from healthy individuals.

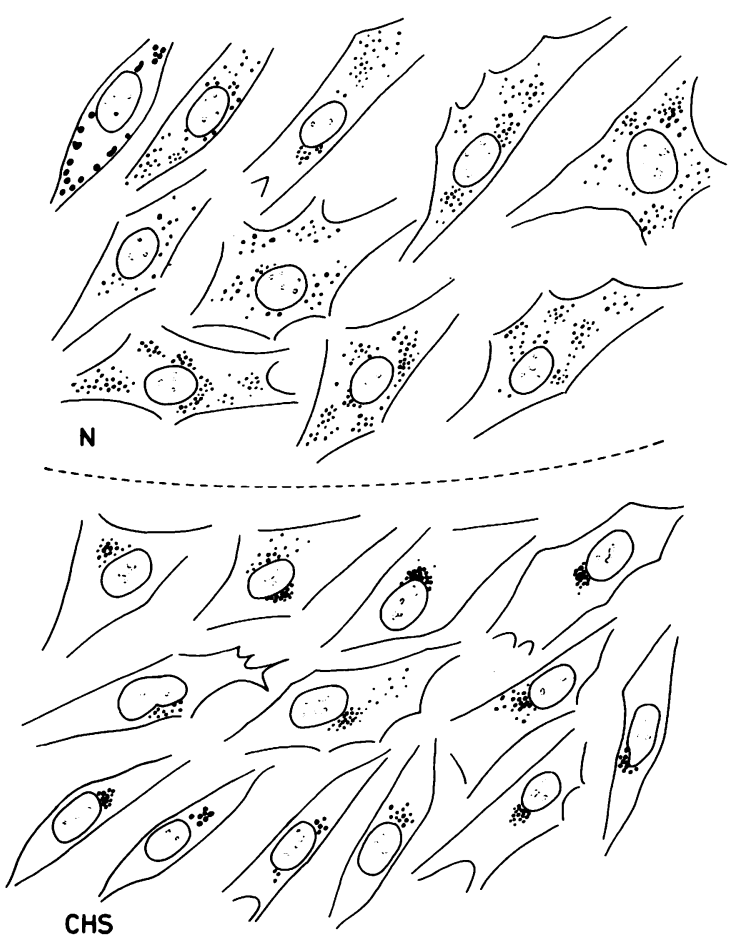

Fig. 3 Drawings of cultured fibroblasts from the normal control $(N)$ and those from the patient with Chediak-Higashi syndrome (CHS). The distribution pattern of lysosomal granules in the cytoplasm is different in each culture.

In the fibroblasts from the control and the mother of the patient, the granules were dispersed throughout the cytoplasm, and also appeared in the long cytoplasmic processes (Figs. 1, 3). On the other hand, the granules in the fibroblasts derived from the patient were clustered adjacent to the nucleus or around the nucleus (Figs. 2, 3). Even after repeated subcultures, the clustered pattern of the granules were observed in the fibroblasts from the patient.

In electron microscopy, the cytoplasm of the fibroblasts contained many membrane-bounded inclusions, 0.1 to $2.0 \mu \mathrm{m}$ in diameter, which were round or oval and had variable contents (Fig. 4a). Ultrastructural differences of the inclusions were not noticed for the fibroblasts from different sources. The inclusions appeared throughout the cytoplasm except for the Golgi area in the fibroblasts from the healthy individuals. In the fibroblasts from the patient, the inclusions appeared around the Golgi apparatus near the nucleus, and almost no inclusions were observed in the peripheral cytoplasm (Fig. 4a). Microtubules and filaments were observed through- 

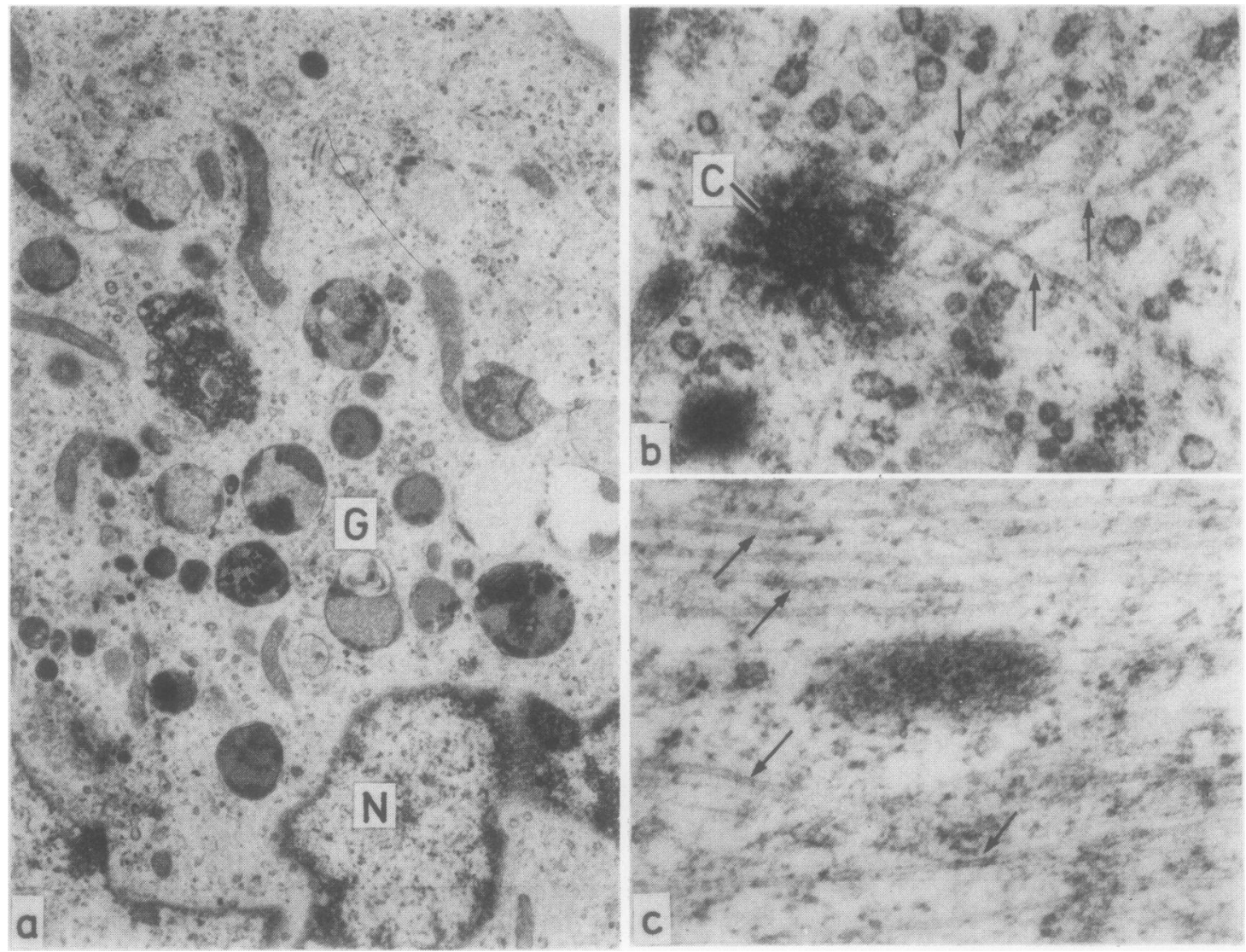

Fig. 4 Fibroblast from the patient with Chediak-Higashi syndrome. Electron microscopy. (a) Perinuclear region. Many inclusions are seen around the Golgi apparatus $(G)$ near the nucleus $(N) \times 8000$. (b) Centriolar region. Microtubules (arrows) are seen around the centriole $(C) \times 40$ 000. (c) Peripheral region. Abundant microtubules are observed (arrows) $\times 60000$.

out the cytoplasm and in the centriolar region of the fibroblasts from the patient and healthy individuals (Fig. 4bc). The shape, amount, and distribution of microtubules were not different for the fibroblasts derived from the different sources.

\section{QUANTITATIVE EVALUATION OF THE}

DISTRIBUTION PATTERN OF THE GRANULES

The differences in the distribution pattern of the granules in the fibroblasts were studied quantitatively as follows. The fibroblasts were divided into four classes according to the width of the perinuclear cytoplasm containing the granules, as shown in Fig. 5. The fibroblasts in which the width was narrower than half the nuclear diameter, equal to the nuclear diameter, twice the nuclear diameter, or wider than twice the nuclear diameter were classified as class $1,2,3$, or 4 , respectively. The percentage $(P i)$ of the fibroblasts of each class cut

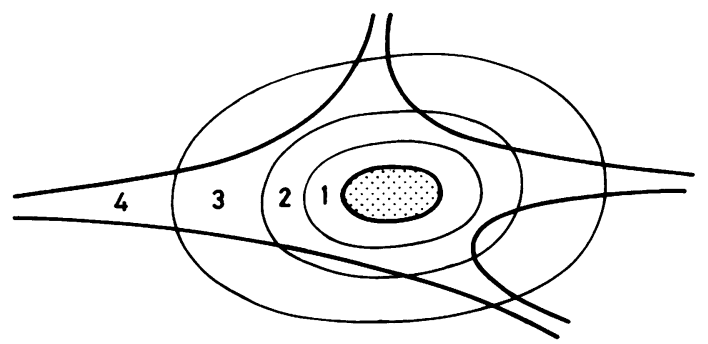

Fig. 5 Diagram of cultured fibroblasts representing the classification. They are classified into four classes (1-4) according to the width of perinuclear cytoplasm containing lysosomal granules. The numbers $(1,2,3$, or 4) show the border of perinuclear cytoplasm with the granules.

of the total fibroblasts counted in each culture was obtained (Table). Furthermore, an index indicating 
Proportion of fibroblasts in each class representing the distribution pattern of lysosomal granules mean ( $\%)$; ranges are in parentheses

\begin{tabular}{llll}
\hline Class & $N$ & CHSM & CHS \\
\hline 1 & $1 \cdot 0(0-2)$ & $0 \cdot 6(0-1)$ & $76 \cdot 0(64-86)$ \\
2 & $13 \cdot 5(5-21)$ & $14 \cdot 0(8-21)$ & $20 \cdot 0(12-28)$ \\
3 & $48 \cdot 0(38-61)$ & $48 \cdot 7(44-53)$ & $4 \cdot 3(2-8)$ \\
4 & $37 \cdot 5(24-56)$ & $36 \cdot 7(29-42)$ & $0 \cdot 3(0-1)$ \\
\hline
\end{tabular}

CHS = patient with Chediak-Higashi syndrome.

CHSM = patient's mother.

$\mathrm{N} \quad=$ normal control.

the distribution pattern of the granules in the fibroblasts for each culture was represented by $\sum_{i=1}^{4}(i-1) P i / 3$. The index becomes 0 if all fibroblasts belong to class 1 , or 100 if all fibroblasts belong to class 4.

As seen in the Table and Fig. 6, in the culture from

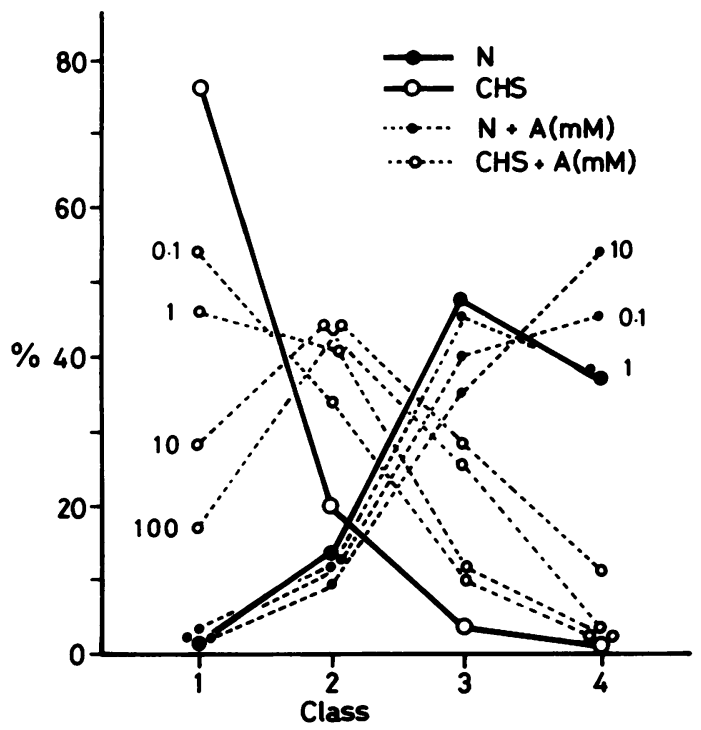

Fig. 6 Proportion of fibroblasts in each class. $N=$ normal control; $\mathrm{CHS}=$ Chediak-Higashi syndrome. Broken lines represent the changes in the distribution pattern after incubation with $0 \cdot 1,1,10$, or $100 \mathrm{mM}$ ascorbic acid $(A)$ in the medium.

the mother and the control, half the fibroblasts were included in class 3 , and the fibroblasts in class 4 were also frequent, whereas those in class 1 and 2 were rare. However, the fibroblasts from the patient were mostly in class 1 , but rare in class 3 and 4 . The index (average) of the distribution pattern of the granules was 75 for the culture from the mother, 74 for the control, and 9.4 for the Chediak-Higashi syndrome (Fig. 7). Thus, the distribution pattern

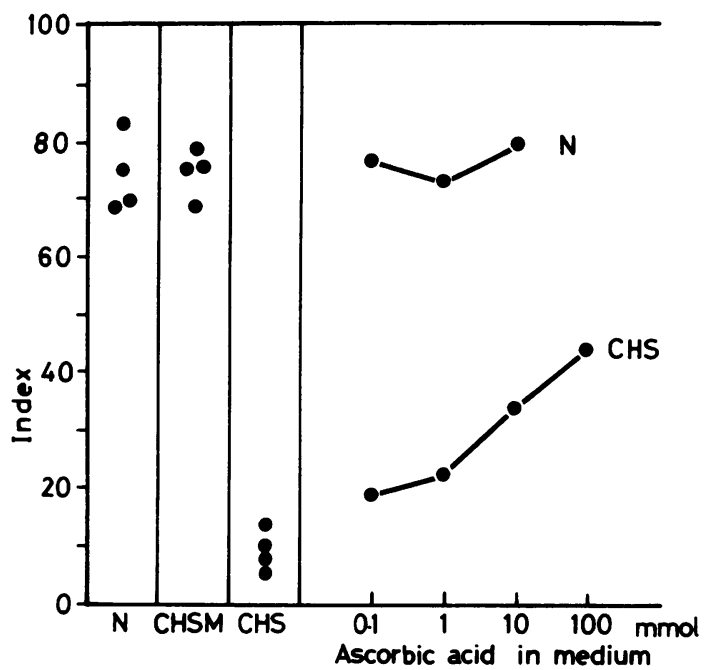

Fig. 7 Index for the distribution pattern of the granules. $N=$ normal; $C H S M=$ mother of the patient with Chediak-Higashi syndrome; CHS = the patient with Chediak-Higashi syndrome. The effect of increasing the concentration of ascorbic acid in the medium on the index is shown on the right.

of the cytoplasmic granules showed quantitatively significant differences between the fibroblasts from the patient with the Chediak-Higashi syndrome and those from the other sources.

\section{Effects of ascorbic acid}

With increasing dose of ascorbic acid, some fibroblasts were rounded on the glass and left from the surface of the glass. After incubation with $100 \mathrm{mM}$ ascorbic açid, about a quarter of the fibroblasts were spreading and the others were rounded on the glass. The cytoplasmic granules, mentioned above, were recognised in the fibroblasts spreading on the glass. The distribution pattern of the granules in the control fibroblasts treated with ascorbic acid was similar to that in the fibroblasts cultured without ascorbic acid (Figs. 6, 7). With increasing dose of ascorbic acid, the fibroblasts from the Chediak-Higashi syndrome showed a decrease of percentage of the cells in class 1 (Fig. 6), the clustered granules tended to spread throughout the cytoplasm, and the index showed an increase (Fig. 7).

\section{Discussion}

Oliver et $a l,{ }^{6}$ who analysed the function of microtubules using cells from normal and beige mice, observed lysosomal granules clustered in the perinuclear area of the cultured fibroblasts from beige mice, and the granules dispersed throughout 
the cytoplasm in the normal fibroblasts. We noticed similar findings in the cultured fibroblasts from a patient with the Chediak-Higashi syndrome and normal individuals. The presence of acid phosphatase in the PAS-positive granules in our cultured fibroblasts indicates that the granules are lysosomal. ${ }^{13}$ These lysosomal granules in the Chediak-Higashi fibroblasts were clustered adjacent to the nucleus, whereas those in the normal fibroblasts were distributed throughout the cytoplasm. Thus, the ChediakHigashi fibroblast cultures were readily distinguished from the others.

It is said that lysosomes arise from the Golgi apparatus and are scattered in the cytoplasm like secretory granules. ${ }^{14}$ It has been known that cellular movement and intracellular migration of secretory granules, organelles, and inclusions depend on the function of microtubules and microfilaments. ${ }^{15}$ However, recent studies concerning the ChediakHigashi syndrome have suggested that impaired bacteriocidal activities and chemotaxis of polymorphonuclear leucocytes, and abnormal migration of concanavalin A receptor complexes on the polymorphonuclear leucocytes are due to abnormal microtubular assembly in the syndrome. ${ }^{16-19}$ Therefore, genetic disorder of the Chediak-Higashi syndrome seems to be included in the intracellular mechanism regulating microtubular assembly. Morphologically it has been reported that no microtubules were detectable in the centriolar region of the polymorphonuclear leucocytes in the ChediakHigashi syndrome, ${ }^{6}$ but microtubules in our ChediakHigashi fibroblasts seemed normal in number and structure. Thus, the cluster of the granules in the fibroblasts from the patient indicate dysfunction of microtubules in the Chediak-Higashi syndrome.

In this study, we used the index to express the distribution pattern of the granules in the fibroblasts, and the index clearly differentiated the ChediakHigashi fibroblasts from the mother's or normal fibroblasts. The index of the Chediak-Higashi fibroblasts rose towards the normal level after treatment with ascorbic acid, which is reflected in improved microtubular function. ${ }^{720}$ Therefore, the index for the distribution pattern of lysosomal granules in cultured fibroblasts is considered to be useful for the study of the impaired function of microtubular assembly in the Chediak-Higashi syndrome; it may also suggest the degree of the disease.

The index in the fibroblasts from the mother, carrier of the abnormal gene, showed a normal level. On the other hand, Danes and Bearn ${ }^{11}$ observed large inclusions in the cultured fibroblasts from the parents of the patient, and recommended cell culture in detecting the carrier. Such inclusions were not shown in our Chediak-Higashi fibroblasts. Abnormally large inclusions shown by them ${ }^{11}$ seem slightly different in stainability from the granules in our fibroblasts. The differences between our fibroblasts and those observed by Danes and Bearn ${ }^{11}$ may be related to differences in the onset of the disease. Their fibroblasts were obtained from a 14-year-old patient, while our patient died 110 days after birth. However, large granules in the cultured fibroblasts from beige mice were prominent in confluent cultures in 10 to 14 days, ${ }^{6}$ and it was suggested that such abnormal granules arose by fusion of developing granules. ${ }^{21}$ We observed the fibroblasts in two days of subculture. The granules in our Chediak-Higashi fibroblasts may become larger in confluent culture.

It is hoped that the fetal genetic diseases can be diagnosed during early pregnancy, ${ }^{1022}$ but the value of amniocentesis in Chediak-Higashi syndrome has not been established. The perinuclear congregation of the lysosomal granules in the fibroblasts of the Chediak-Higashi syndrome persists during repeated subculture. Cultured cells usually contain such granules. Therefore, the amniotic cell culture, in which fibroblast-like cells and epithelial cells grow, ${ }^{23}$ is also considered to be useful in prenatal diagnosis of the syndrome.

\section{References}

${ }^{1}$ Chediak MM. Nouvelle anomalie leucocytaire de caractère constitutionnel et familial. Rev Hematol 1952;7:362-7.

${ }^{2}$ Higashi $\mathrm{O}$. Congenital gigantism of peroxidase granulefirst case ever reported of qualitative abnormality of peroxidase. Tohoku J Exp Med 1954;59:315-32.

${ }^{3}$ Douglas SD, Blume RS, Wolff SM. Fine structural studies of leukocytes from patients and heterozygotes with Chediak-Higashi syndrome. Blood 1969;33:527-40.

${ }^{4}$ Lutzner MA, Tierney JH, Benditt EP. Giant granules and widespread cytoplasmic inclusions in a genetic syndrome of Aleutian mink: an electron microscopic study. Lab Invest 1966;14:2063-79.

${ }^{5}$ Bennett JM, Blume RS, Wolff SM. Characterisation and significance of abnormal leukocyte granules in the beige mouse: a possible homologue for Chediak-Higashi Aleutian trait. $J$ Lab Clin Med 1969;73:235-43.

- Oliver JM, Krawiec JA, Berlin RD. Carbamylcholine prevents giant granule formation in cultured fibroblasts from beige" (Chediak-Higashi) mice. J Cell Biol 1976; 69:205-10.

'Boxer LA, Watanabe AM, Rister M, Besch HR Jr, Allen J, Baehner RL. Correction of leukocyte function in Chediak-Higashi syndrome by ascorbate. $N$ Engl $J$ Med 1976;295:1041-5.

${ }^{8}$ Leory JG, De Mars RI. Mutant enzymatic and cytological phenotypes in cultured human fibroblasts. Science 1967;157:804-6.

- Wiesman UN, Herschkowitz NN. Studies on the pathogenic mechanism of I-cell disease in cultured fibroblasts. Pediatr Res 1974;8:865-70.

${ }^{10}$ Abe K, Matsuda I, Arashima S, Mitsuyama T, Oka Y, Ishikawa M. Ultrastructural studies in fetal I-cell disease. Pediatr Res 1976;10:669-76. 
11 Danes BS, Bearn AG. Cell culture and the ChediakHigashi syndrome. Lancet 1967; ii:65-7.

12 Tanakawa N. Anakura M, Iizuka S, et al. A case of the Chediak-Higashi syndrome (in Japanese). Shonikarinsho $1980 ; 33$ :1531-6.

${ }^{13}$ Dingle JT, ed. Lysosomes in biology and pathology, 3. North-Holland, American Elsevier, 1973.

${ }^{14}$ Novikoff AB, Essner E, Quintana N. Golgi apparatus and lysosomes. Fed Proc 1964;23:1010-22.

${ }^{15}$ Stossel TP. Phagocytosis. N Engl J Med 1974;290:717-23.

${ }^{16}$ Clark R, Kimball H. Defective granulocyte chemotaxis in the Chediak-Higashi syndrome. J Clin Invest 1971; 50:2645-52.

17 Stossel TP, Root RK, Vaughan M. Phagocytosis in chronic granulomatous disease and the Chediak-Higashi sydrome. N Engl J Med 1972;286:120-3.

${ }^{18}$ Wolff SM, Dale DC, Clark RA, Root RK, Kimball HR. The Chediak-Higashi syndrome: studies of host defenses. Ann Intern Med 1972;76:293-306.

${ }^{19}$ Oliver JM. Impaired microtubule assembly in ChediakHigashi syndrome neutrophils correctable by cyclic GMP and cholinergic agents. Am J Pathol 1976;85: 395-418.
${ }^{20}$ Sandler JA, Gallin JI, Vaughan M. Effects of serotonin, carbamylcholine and ascorbic acid on leukocyte cyclic GMP and chemotaxis. J Cell Biol 1975;67:480-4.

${ }^{21}$ Davis WC, Spicer SS, Greene WB. Padgett GA. Ultrastructure of bone marrow granulocytes in normal mink and mink with the homolog of the Chediak-Higashi trait of human. I. Origin of the abnormal granules present in the neutrophils of mink with C-HD trait. Lab Invest $1971 ; 24: 303-17$.

${ }^{22}$ Simpson NE, Daillaire L, Miller JR, Siminovich L, Hamaerton JL, Miller J, McKeen C. Prenatal diagnosis of genetic disease in Canada: report of a collaborative study. Can Med Assoc J 1976;23:739-48.

${ }^{23}$ Gerbie AB, Melancon SB, Ryan C, Nadler HL. Cultivated epithelial-like cells and fibroblasts from amniotic fluid: their relationship to enzymatic and cytologic analysis. Am J Obstet Gynecol 1972;114:314-20.

Requests for reprints to: Dr K Abe, Department of Anatomy, Hokkaido University School of Medicine, Sapporo, 060 Japan. 